\title{
Oral Malignant Melanoma Initially Misdiagnosed as a Racial Pigmentation: A Case Report
}

\author{
Carla Patrícia Martinelli-Kläy a, b Marcel Leandro Laporte ${ }^{a}$ \\ Celso Ricardo Martinelli ${ }^{b}$ Celso Martinelli ${ }^{b}$ Tommaso Lombardi ${ }^{a}$ \\ ${ }^{a}$ Oral Medicine and Oral Maxillofacial Pathology Unit, Division of Oral Maxillofacial Surgery, \\ Department of Surgery, Geneva University Hospitals and Faculty of Geneva, Geneva, \\ Switzerland; ${ }^{\mathrm{b} C e n t r e}$ for Diagnosis and Treatment of Oral Diseases, Ribeirão Preto, Brazil
}

\section{Key Words}

Oral mucosa - Oral malignant melanoma - Racial pigmentation · Differential diagnosis . Immunohistochemistry

\section{Abstract}

Oral malignant melanoma (OMM) is rare, representing less than $0.5 \%$ of all oral malignancies. The most affected sites are the palate and the maxillary gingiva. Histological examination is important to establish the diagnosis of any suspicious pigmented lesion in the oral cavity, mainly if a precise clinical diagnosis is not possible. We present one case of OMM that was initially diagnosed as a racial pigmentation elsewhere 2 years earlier. Clinical examination showed multiple macules and nodules located on the hard and soft palate, gingiva and superior alveolar mucosa. These lesions were painless and presented a color variation going from dark blue to black. Histological analysis showed sheets and nests of atypical melanocytes displaying a range of shapes such as plasmacytoid, epithelioid, and round cells, located in the superficial corium extending to the deep tissues. A few tumor cells contained variable amounts of melanin. There was no invasion of blood vessels or nerve fibers. Immunohistochemical analysis revealed that the neoplastic cells were positive for HMB-45, melan-A, S-100 and negative for AE1/AE3, confirming the diagnosis of melanoma. The Ki- 67 labeling index was around $25 \%$. The patient refused any treatment and died 11 months later. 
Martinelli-Kläy et al.: Oral Malignant Melanoma Initially Misdiagnosed as a Racial Pigmentation: A Case Report

\section{Introduction}

Melanoma is a malignant condition that arises from melanocyte cells. It develops most of the time on the skin [1-3]; however, other parts of the body could be involved, including the oral cavities $[4,5]$.

Oral malignant melanoma (OMM), unlike cutaneous melanoma, is considered a rare lesion with an incidence of $0.4-1.8 \%$ of all melanomas and $0.5 \%$ of all oral malignancies [6, 7]. The etiology of OMM is still not defined, contrarily to cutaneous melanoma for which sunlight and genetic background are considered etiologic factors [2]. The most frequently affected oral sites are the palate and the maxillary gingiva; other less frequently affected sites include the labial and buccal mucosa as well as the tongue $[1,3,8-12]$. The average age of patients diagnosed with OMM ranges from 40 to 60 years $[1,13]$. There is no sex predilection [9]. Black Africans, Japanese, and Native Americans are more commonly affected than the European population $[1,12,13]$.

Histologically, melanomas are characterized by proliferation of malignant melanocytes arranged along the basal layer, having the potential for tissue invasion and metastasis. These

Fig. 1. Clinical aspect of OMM. a Ulcerated nodule and irregular macules on the hard palate extending to the soft palate. $\mathbf{b}$ Nodule located on the gingiva and alveolar mucosa (upper left lateral incisor region). The color of the lesions varied from dark blue to black.
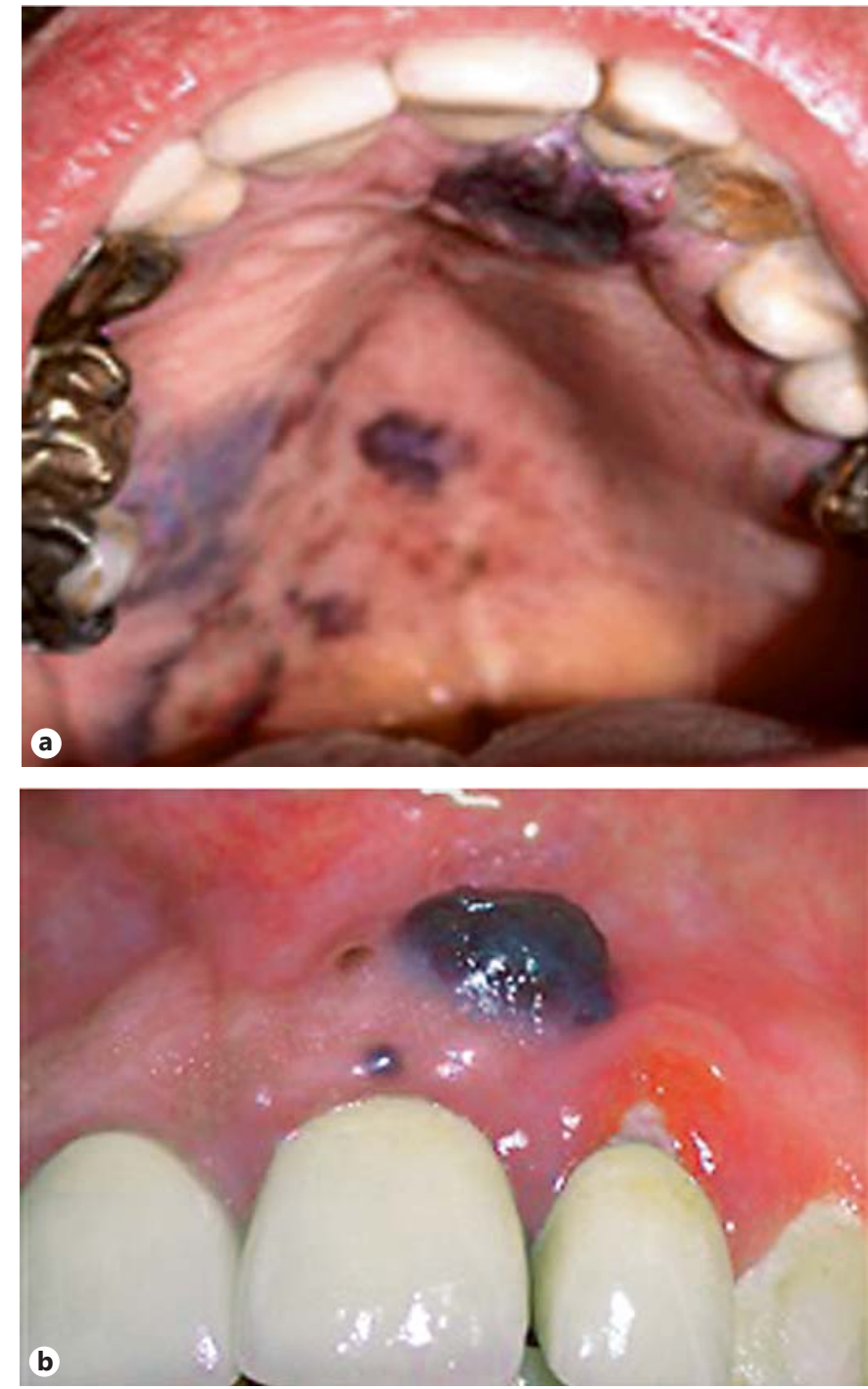
cells show a considerable pleomorphism, with a wide range of shapes, such as round to spindled, plasmacytoid, clear cell and epithelioid, and have a solid, alveolar, organoid or pagetoid pattern. Their nuclei are irregular and sometimes hyperchromatic $[3,6,12]$. Invasion of blood vessels and nerve fibers is common. Necrosis, lack of an inflammatory reaction, and not always detectable mitotic activity are other histological features of this disease [3].

The purpose of this report is to describe a case of OMM and to emphasize the importance of histological examination.

\section{Case Report}

An 80-year-old woman was referred to our clinic for evaluation and treatment of a pigmented oral lesion. She had a history of pigmentations diagnosed elsewhere 2 years earlier as racial pigmentation. Intraoral examination showed multiple lesions represented by macules and nodules on the hard and soft palate, superior alveolar mucosa and gingiva (fig. 1a, b). They were irregular and their color varied from dark blue to black. The size of the lesions ranged from 0.4 to $3.5 \mathrm{~cm}$ in dimension. One of them was an ulcerated nodule causing a little discomfort. There were no palpable lymph nodes of the head and neck regions. A biopsy, which included the free and attached gingiva and alveolar mucosa was performed and submitted to histopathological analysis.

Histological examination (fig. 2a, b) showed sheets and nests of melanocytes, some with round, plasmacytoid or epithelioid morphology in the superficial corium extending to the

Fig. 2. Histological examination. a Sheets and nests of atypical melanocytes located in the corium. HE. Final magnification $\times 100$. The overlying epithelium was atrophic and not pigmented. b Atypical melanocytes arranged in an alveolar pattern, some present round nuclei of various sizes, occupying nearly the entire cell cytoplasm and prominent nucleoli. A few tumor cells contain dense cytoplasmic melanin pigment (arrow). HE. Final magnification $\times 400$.
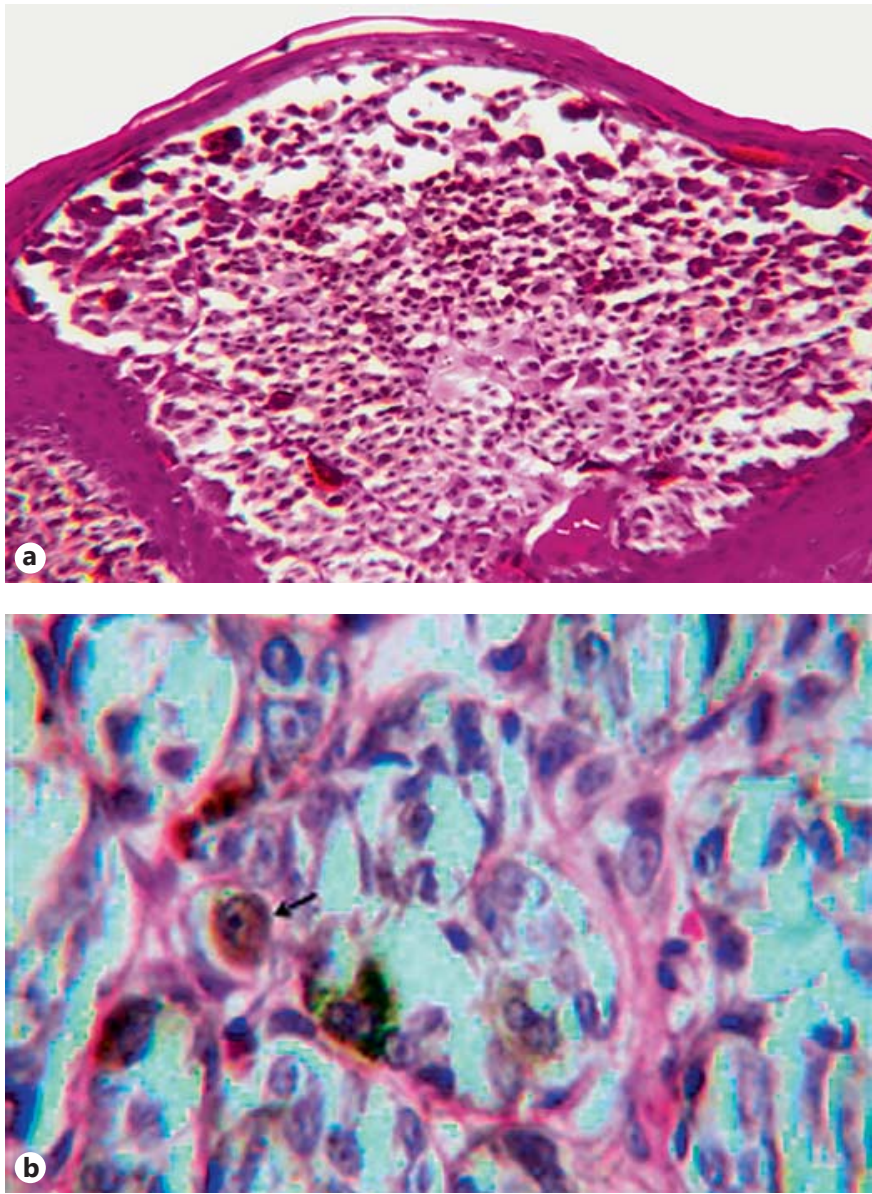

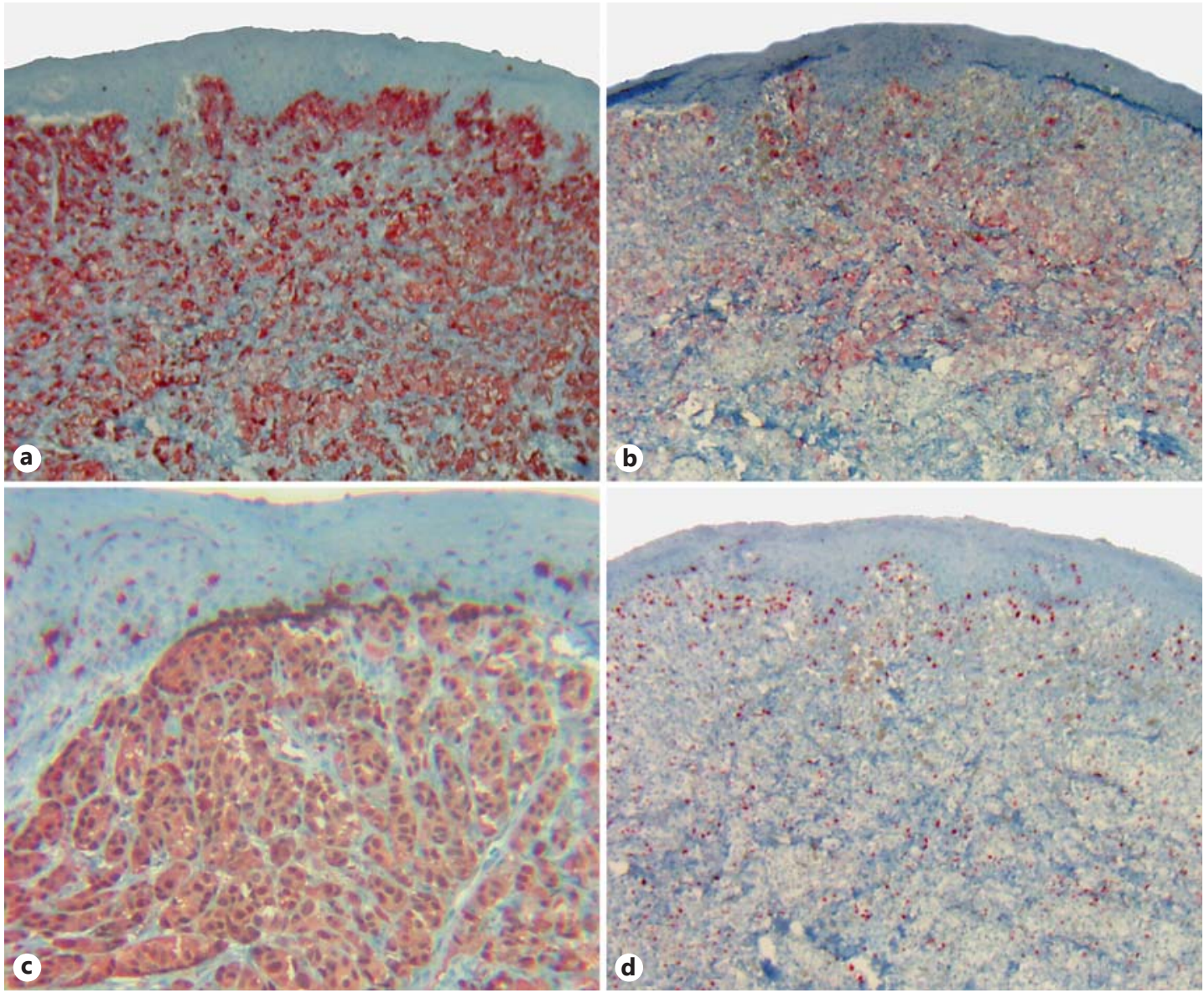

Fig. 3. Immunohistochemical analysis. Neoplastic melanocytes positive for HMB-45 (a), melan-A (b), S-100 (c; final magnification $\times 400$ ) and Ki-67 (d), which shows a labeling index of around 25\%. a, b, d Final magnification $\times 100$.

deep tissues. Some of them presented round nuclei of various sizes, occupying nearly the entire cell cytoplasm and prominent nucleoli. A few tumor cells contained variable amounts of melanin. The overlying epithelium was atrophic and did not present melanocytes. There was no invasion of blood vessels or nerve fibers. Immunohistochemistry showed tumor cells positive for HMB-45 (fig. 3a), melan-A (fig. 3b), S-100 (fig. 3c), Ki-67 (fig. 3d) and negative for AE1/AE3 (not shown), confirming the diagnosis of melanoma. The Ki-67 labeling index was around $25 \%$.

The patient refused a complete workup examination (i.e. CT scan, MRI, etc.) as well as any type of treatment and died 11 months later.

\section{Discussion}

We presented a case of OMM which was initially misdiagnosed as a probable racial pigmentation elsewhere 2 years earlier. This diagnosis was made by taking only the clinical aspect into account and without performing histological analyses. Acquired and small congenital nevi show a high frequency of BRAF mutations [14]. In cutaneous melanoma, 
BRAF mutation is present in about $50 \%$ of the cases [15]. Nevertheless, there is no report in the literature about the potential malignant transformation rate of oral melanocytic nevi or other pigmented lesions [16]. In our case, we cannot establish if the melanoma arose de novo or from a preexistent benign pigmented lesion or precursor, since a biopsy was not carried out. Therefore, all suspicious lesions presenting an asymmetric, irregular form, with variation in color and diameter should be investigated in order to obtain a definitive diagnosis $[3,17]$. The differential diagnosis for pigmented lesions includes tattoos, oral melanotic macule, racial pigmentation, nevi, melanoacanthoma, melanoma and systemic diseases such as polyostotic fibrous dysplasia, neurofibromatosis, multiple endocrine neoplasia syndrome, PeutzJeghers syndrome, and Addison's disease $[9,13,17,18]$.

Initially, OMM appears as an asymptomatic lesion characterized by a flat macule, or a slightly elevated lesion that may later become elevated and develop signs and symptoms such as swelling, ulceration, bleeding, pain and tooth mobility $[6,13]$. According to the literature, swelling is considered the main reason for consultation, followed by bleeding, ill-fitting dentures, pain, increased mobility of teeth and delayed healing of extraction sockets $[6,8,9]$.

OMM occurs in adults of all ages, but it is most common in the elderly. Aguas et al. [9], when studying 187 cases, showed an average age of 67.5 years, and an unusual case of a 9 -year-old patient was described by Lorenço et al. [10]. There is no agreement in the literature concerning the ratio of the patients' gender, having no sex predilection or presenting a slight predilection for men or sometimes for women $[7,9,10]$.

Histologically, the tumor cells show a considerable pleomorphism, with a wide range of shapes and patterns that could mimic other tumors such as lymphomas, poorly differentiated carcinomas, neuroendocrine carcinomas, sarcomas, and germ-cell tumors [19]. In addition, more rarely, the melanomas may be nonpigmented, causing difficulties in clinical diagnosis and are typically diagnosed at a later stage $[2,6,9]$. Therefore, the use of immunohistochemical staining is an important tool to help distinguish melanomas from other malignancies.

The helpful markers of melanoma diagnosis are S-100, HMB-45, melan-A, and tyrosinase [20-22]. For this case, we used S-100, HMB-45 and melan-A.

S-100 is very sensitive (around $97-100 \%)$ but not very specific (75-87\%) [21]. Some carcinomas, sarcomas (such as rhabdomyosarcomas, leiomyosarcomas, and peripheral nerve sheath tumors), and Langerhans cell histiocytosis can be positive for S-100. HMB-45 and melan-A are considered more specific markers. HMB-45 recognizes a type I membrane-bound melanosomal protein called PMEL17 or gp100 protein. The analysis of HMB-45 and Ki-67 antibodies can be useful for distinguishing primary cutaneous melanomas from some nevi. Melan-A is observed in melanosomes and the endoplasmic reticulum of melanocytes [22]. $\mathrm{Ki}-67$ is a marker of tumor cell proliferation, which is commonly used as a complement to distinguish benign nevi from melanoma. With less than $5 \%$ of positive cells, the lesion is considered as benign nevi, while with $13-30 \%$ of positive cells, it is considered as malignant melanoma [21]. In our case, we found about $25 \%$ of Ki-67-positive cells.

The multi-cytokeratin $(A E 1 / A E 3)$ antibody is reported to be characteristic of epidermal cells. It is useful to distinguish melanoma from carcinoma and other S-100-positive malignancies [21].

Many predictors of prognostics have been proposed in the literature on OMM such as age, clinical stage, depth of invasion and vascular invasion $[4,9,10,23]$. However, because of the rarity of this disease, consensus criteria have not yet been established [4]. According to the microstaging classification by Prasad et al. [24], which is based on the invasion of the tissue within the mucosa, level I is defined as melanoma in situ, level II as melanoma invading up to the lamina propria and level III as melanoma with deep tissue invasion. Our case was classified as level III because the malignant melanocytes reached the periosteum. However, there 
was no invasion of blood vessels and nerve fibers. Melanoma has an initial phase named radial or horizontal growth, which is characterized by a laterally spreading growth of malignant melanocytes only within the epithelium, representing the in situ stage. Later, in the vertical growth phase, there is an invasion of the underlying tissues $[8,9]$. OMM is normally diagnosed in the vertical growth pattern, which may explain its very poor prognosis.

In this case, the patient refused any type of treatment and died 11 months after the diagnosis. It has been shown that early identification of the melanoma and its treatment by radical surgery comprise the most important treatment strategy $[11,25,26]$. An association with adjuvant radiation can also be performed. In cases of metastatic diseases, chemotherapy should be considered with the aim of improving the survival of patients with head and neck melanoma [26].

Due to the deadly behaviour of OMM, showing a poor prognosis with a 5-year survival rate of $15 \%$, all focally pigmented lesions and most diffusely pigmented lesions require a biopsy for diagnosis. The early identification of OMM is the key for improving successful treatment.

\section{Statement of Ethics}

Written informed consent was obtained from a family member.

\section{Disclosure Statement}

The authors have no conflicts of interest to disclose.

\section{References}

1 Garzino-Demo P, Fasolis M, Maggiore GM, Pagano M, Berrone S: Oral mucosal melanoma: a series of case reports. J Craniomaxillofac Surg 2004;32:251-257.

2 Neville BW, Damm DD, Allen CM, Chi AC: Epithelial pathology; in Oral and Maxillofacial Pathology, ed 4. St. Louis, Saunders, 2015, pp 401-421.

-3 Hicks MJ, Flaitz CM: Oral mucosal melanoma: epidemiology and pathobiology. Oral Oncol 2000;36:152-169.

$\checkmark 4$ Kerr EH, Hameed O, Lewis JS Jr, Bartolucci AA, Wang D, Said-Al-Naief N: Head and neck mucosal malignant melanoma: clinicopathologic correlation with contemporary review of prognostic indicators. Int J Surg Pathol 2012;20:37-46.

5 Femiano F, Lanza A, Buonaiuto C, Gombos F, Di Spirito F, Cirillo N: Oral malignant melanoma: a review of the literature. J Oral Pathol Med 2008;37:383-388.

6 Meleti M, Leemans CR, Mooi WJ, Vescovi P, Van der Waal I: Oral malignant melanoma: a review of the literature. Oral Oncol 2007;43:116-121.

7 de-Andrade BA, Toral-Rizo VH, León JE, Contreras E, Carlos R, Delgado-Azañero W, Mosqueda-Taylor A, de-Almeida OP: Primary oral melanoma: a histopathological and immunohistochemical study of 22 cases of Latin America. Med Oral Patol Oral Cir Bucal 2012;17:383-388.

-8 Gondak RO, Jorge RS, Jorge J, Lopes MA, Vargas PA: Oral pigmented lesions: clinicopathologic features and review of the literature. Med Oral Patol Oral Cir Bucal 2012;17:919-924.

-9 Aguas SC, Quarracino MC, Lence AN, Lanfranchi-Tizeira HE: Primary melanoma of the oral cavity: ten cases and reviewed of 177 cases from literature. Med Oral Patol Oral Cir Bucal 2009;14:265-271.

-10 Lorenço SV, Sangüeza A, Sotto MN, Bologna SB, Di Giacomo TB, Coutinho-Camillo CM, Buim ME, Silva SD, Landman G, Soares FA, Simonsen MM: Primary oral mucosal melanoma: series of 35 new cases from South America. Am J Dermatopathol 2009;31:323-330.

11 Mücke T, Hölzle F, Kesting MR, Loeffelbein DJ, Robitzky LK, Hohlweg-Majert B, Tannapfel A, Wolff KD: Tumor size and depth in primary malignant melanoma in the oral cavity influences survival. J Oral Maxillofac Surg 2009;67:1409-1415.

12 Meleti M, Leemans CR, Mooi WJ, van der Waal I: Oral malignant melanoma: the Amsterdam experience. J Oral Maxillofac Surg 2007;65:2181-2186. 
13 Manganaro AM, Hammond HL, Dalton MJ, Williams TP: Oral melanoma: case reports and review of the literature. Oral Surg Oral Med Oral Pathol Oral Radiol Endod 1995;80:670-676.

14 Takata M, Saida T: Genetic alterations in melanocytic tumors. J Dermatol Sci 2006;43:1-10.

15 Long GV, Menzies AM, Nagrial AM, Haydu LE, Hamilton AL, Mann GJ, Hughes TM, Thompson JF, Scolyer RA, Kefford RF: Prognostic and clinicopathologic associations of oncogenic BRAF in metastatic melanoma. J Clin Oncol 2011;29:1239-1246.

16 Meleti M, Mooi WJ, Casparie MK, van der Waal I: Melanocytic nevi of the oral mucosa - no evidence of increased risk for oral malignant melanoma: an analysis of 119 cases. Oral Oncol 2007;43:976-981.

17 Kuffer R, Lombardi T, Husson-Bui C, Courrier B, Samson J: Troubles de la pigmentation et tumeurs pigmantaires; in La Muqueuse Buccale de la Clinique au traitement, ed 1. Paris, MED'COM, 2009, pp 139-141.

18 Alawi F: Pigmented lesions of the oral cavity: an update. Dent Clin North Am 2013;57:699-710.

-19 Banerjee SS, Harris M: Morphological and immunophenotypic variations in malignant melanoma. Histopathology 2000;36:387-402.

-20 Viray H, Bradley WR, Schalper KA, Rimm DL, Gould Rothberg BE: Marginal and joint distributions of S100, HMB-45, and Melan-A across a large series of cutaneous melanomas. Arch Pathol Lab Med 2013;137:10631073.

21 Ohsie SJ, Sarantopoulos GP, Cochran AJ, Binder SW: Immunohistochemical characteristics of melanoma. J Cutan Pathol 2008;35:433-444.

-22 Ordóñez NG: Value of melanocytic-associated immunohistochemical markers in the diagnosis of malignant melanoma: a review and update. Hum Pathol 2014;45:191-205.

-23 Jethanamest D, Vila PM, Sikora AG, Morris LG: Predictors of survival in mucosal melanoma of the head and neck. Ann Surg Oncol 2011;18:2748-2756.

24 Prasad ML, Patel SG, Huvos AG, Shah JP, Busam KJ: Primary mucosal melanoma of the head and neck: a proposal for microstaging localized, Stage I (lymph node-negative) tumors. Cancer 2004;100:1657-1664.

25 Mendenhall WM, Amdur RJ, Hinerman RW, Werning JW, Villaret DB, Mendenhall NP: Head and neck mucosal melanoma. Am J Clin Oncol 2005;28:626-630.

-26 Moreno MA, Hanna EY: Management of mucosal melanomas of the head and neck: did we make any progress? Curr Opin Otolaryngol Head Neck Surg 2010;18:101-106. 